\title{
Possible Caveats of Ultra-Short Heart Rate Variability Reliability: Insights from Recurrence Quantification Analysis
}

Hiago Murilo Melo

Federal University of Santa Catarina (UFSC)

Mariana Cardoso Melo ( $\square$ mcmelo@unifesp.br)

Federal University of São Paulo (UNIFESP)

Roger Walz

Federal University of Santa Catarina (UFSC)

\section{Emílio Takase}

Federal University of Santa Catarina (UFSC)

Jean Faber

Federal University of São Paulo (UNIFESP)

\section{Research Article}

Keywords: Heart rate variability, Ultra-short term HRV, Recurrence quantification analysis, Autonomic nervous system, rMSSD

Posted Date: July 15th, 2021

DOl: https://doi.org/10.21203/rs.3.rs-720309/v1

License: (c) (1) This work is licensed under a Creative Commons Attribution 4.0 International License. Read Full License 


\title{
Possible Caveats of Ultra-Short Heart Rate Variability Reliability: Insights from Recurrence Quantification Analysis
}

\author{
Hiago Murilo Melo ${ }^{1,2,4}$, Mariana Cardoso Melo ${ }^{5}$, Roger Walz ${ }^{2,3,4}$, Emílio Takase $^{1}$ and Jean Faber ${ }^{5}$ \\ 1.Brain Education Laboratory (LEC), Department of Psychology, Federal University of Santa Catarina, Florianopolis, Brazil \\ 2.Postgraduate Program in Neurosciences, Federal University of Santa Catarina, Florianopolis, Brazil \\ 3.Postgraduate Program in Medical Sciences, Federal University of Santa Catarina, Florianopolis, Brazil \\ 4.Center of Applied Neuroscience (CeNAp), Department of Clinical Medicine, Federal University of Santa Catarina, Florianopolis, Brazil \\ 5.Escola Paulista de Medicina (EPM) - Federal University of São Paulo (UNIFESP), Dept. of Neurology and Neurosurgery, Lab. of \\ Neuroengineering and Neurocognition (LaNN), São Paulo, Brazil
}

\begin{abstract}
The heart rate variability (HRV) is the difference between consecutive $R-R$ intervals of heartbeats measured in milliseconds. HRV indices represent the role of sympathetic and parasympathetic autonomic branches. Even though HRV is considered an indirect biomarker of Autonomic Nervous System, there are not yet standardized protocols providing reliable clinical measures. One of the reasons is because HRV techniques requires long recording periods. There are attempts of decreasing the required recording, such as the strategy of ultrashort HVR recording (< one minute), which could make the utilization of the technique easier. However, there is little published about its reliability. This work proposes a method to evaluate the reliability of ultra-short HVR based in Poincare map and Recurrence Quantification Analysis, well known methods to assess nonlinear and dynamic information from a system, in order to verify the reliability of the use of ultra-short term HRV. Then, these results was compared with the classical HRV coefficients, such as rMSSD, recorded from subjects in spontaneous breathing and also, in controlled breathing protocols. As a conclusion, using the proposed methods, we were able to show the discrepancy between the segments of interest, both on mean and in variance, explained in the analysis of main components.
\end{abstract}

Keywords - Heart rate variability; Ultra-short term HRV; Recurrence quantification analysis; Autonomic nervous system; rMSSD.

\section{INTRODUCTION}

Heart rate variability (HRV) represents a variation between consecutive R-R intervals of heartbeats measured in milliseconds [1]. The fluctuations of HRV are controlled by homeostatic systems, including baroreceptors, respiration, thermoregulation. Also, the cardiovascular system is influenced by sympathetic and parasympathetic autonomic branches [2]. The quantitative analysis of HRV can be performed in time and frequency, providing indices related to the sympathetic and parasympathetic autonomic axis [3]. Several studies report that clinical disorders like obesity, diabetes, cardiovascular diseases, psychiatric disorders present impairment on HRV indices, indicating an autonomic dysfunction [4-8]. Thus, HRV is considered a non-invasive biomarker of the autonomic nervous system (ANS) suitable for clinical and health applications [5-6, 9-11]. Although clinical findings have already been reported by groups of researchers [2-5], HRV assessment is not part of the clinical routine due to the lack of standardized protocols compatible with the clinical setting.

HRV analysis consists of the measure of successive R-R or N-N peaks from electrocardiogram (ECG). This temporal series, also known as tachogram, can be analyzed commonly through frequency or time domain strategies. In the frequency domain, it can be extracted the High Frequency (HF) and Low Frequency (LF) indices from the tachogram, in which HF correspond only to indirect vagal modulation, and LF indices correspond indirectly to a mixed response of both vias (sympathetic and vagal) [12].

Recently, there has been renewed interest in the improvement of methods for HRV recording to refine clinical suitability [13-15]. The gold-standard methods (i.e., electrocardiogram, ECG) for R-R intervals recording presents high correlation coefficients to R-R intervals derived from wireless heart rate modules [13], reducing the financial and operational cost of the HRV technique. To obtain reliable HRV data, it is recommended recordings lengths from five minutes to 24 hours [1-3]. Recent studies show some HRV indices that present good correlations between gold-standard time length (5-minutes) and one-minute time epochs proposing an ultra-short term HRV recording ( $<1$-minute) [14-16]. The results report that Root-Mean-Square of the Successive Normal Sinus R-R interval difference (rMSSD) is the adequate parameter for accessing HRV from ultra-short recordings, while other time or frequency domain indices require longer recording periods [14-16].

There is little published data on the reliability of ultrashort HRV data applied to clinical sample or experimental conditions, as most studies involve healthy samples or are recorded at rest. The currents evidence suggest that ultra- 
short-term rMSSD: a) is sensitive to physical-training induced changes in cardiac autonomic tonus of elite athletes [17-18]; b) is associated to mental stress and emotional frustration perceptions after cognitive workload [19-20]; c) can be used efficiently for autonomic evaluation in diabetes mellitus patients [21]. Nevertheless, ultra-short-term rMSSD reliability remains unclear and many investigations have adopted ultra-short analysis without questioning its validity, there is a clear lack of rigorous methods to identify indices for ultra-short recording [22]. So, other methods of HRV indices extraction could be used to better comprehension of R$\mathrm{R}$ intervals dynamic across distinct recording lengths.

Non-linear strategies such as Poincaré Map (PM) and Recurrence Quantification Analysis (RQA) have been applied to assess dynamics related to ECG and HVR. PM has been used to detect abnormal dynamics of cardiac repolarization [23]; to evaluate dynamic autonomic modulation during general anesthesia induction [24]. There are attempts of using RQA for analysis of heart rate variability and respiratory flow series in patients on weaning trials [25]; heart rate dynamics in young patients with diabetes mellitus [26].

RQA is used for quantification of the number of recurrences of a dynamical system and its duration presented by its phase space trajectory [27]. PM is characterized as a discrete dynamical system that represents the continuous periodic flow of another system [28]. Thus, due to the capability of RQA and Poincaré Map to assess nonlinear and dynamic information from a system, we will apply both strategies to verify the reliability of ultra-short term HRV. Then, the results will be compared with the classical HRV coefficients, such as rMSSD, recorded from subjects in spontaneous breathing and also, in controlled breathing protocols.

\section{Materials AND METHODS}

To evaluate the reliability of ultra-short-term heart rate variability, we performed the HRV analysis by evaluating the tachogram characteristics from two groups, $\mathrm{n}=20$ subjects under controlled breathing and the same subjects under spontaneous breathing, by using Poincaré Map coefficients SD1 and SD2, through six coefficients from Recurrence Quantification Analysis, that are: Recurrence Rate (RR), Determinism (DET), Entropy (ENT), Maximal diagonal line length (Lmax), Laminarity (L), Trapping Time (TT). Also, it will be obtained the classical rMSSD coefficient from HVR signal.

\section{A. Participants}

The sample of this study was composed of 20 participants (all male) aged between 18 and 28 years old $(\mathrm{M}=23.65 \pm$ 3.24). The following exclusion criteria were applied to the sample: (a) Do not follow the pace of the breathing pacer (6 breaths / $\mathrm{min}$ ) satisfactorily or feel discomfort when performing this breathing rhythm; (b) Diagnostic of cardiac dysfunction; (c) Do not drink alcohol (24h before) or caffeine (3h before) drinks before the experiment. This study was approved by a local Ethics Committee, and was conducted according to the Helsinki Declaration.

\section{B. Physiologic Recording and HVR analysis}

PolarH7 heart rate monitor (Polar, Finland) was used for the acquisition of RR intervals (iRR, in ms). All acquisitions have occurred in the period between 9 and 12 hours in a well illuminated and quiet room. The resting baseline was made in sitting position, followed by a breathing paced condition (6 breaths/min), both periods with 6-minute length. To reduce the effects of autonomic adaptation to body posture, the participant received the guidelines of the experimental protocol, signed the consent form and performed a test record in the sitting position

To evaluate different periods of time, four fragments were randomly selected, in which the first is 6-minute length, the second of 3-minute, third with 2-minute, and last with oneminute. Each fragment was sorted 100 times to minimize the bias of selection of the evaluation periods.

\section{Poincaré Map}

The Poincaré Maps was generated by scatter plots given by the past R-R intervals (RRt-n) against the present R-R intervals (RRt). This is done qualitatively by studying cluster shapes and quantitatively using cluster deviations SD2 and $\mathrm{SD} 1$, which represent the major axis and the minor axis of an ellipse, respectively. SD2 quantifies the point distribution across the line of identity (LOI), and SD1 indicates the point of distribution across the perpendicular line to LOI.

\section{Recurrence Quantification Analysis}

RQA is based on the evaluation of dot-structures presented on the Recurrence Plot (RP) $\boldsymbol{R}_{i, j}$ through different nonlinear factors [26]. Each dot $(i, j)$ of the RP is calculated according to the recurrence rule, described by $\boldsymbol{R}_{i, j}=1$, if $\| \vec{x}_{i}-$ $\vec{x}_{j} \| \leq \varepsilon$, and $\boldsymbol{R}_{i, j}=0$ otherwise; where $\|\cdot\|$ is the Euclidian distance metric, $\varepsilon$ is the recurrence threshold and $\vec{x}_{k}$ are vectors pointing to the amplitude coordinates in the phase space $\left(t_{k}, t_{k+\tau}, \ldots, t_{k+(m-1) \tau}\right)$. As the columns and rows of a $\mathrm{RP}$ correspond to temporal coordinates of the time series, the elements of the matrix indicate each moment that a relevant 
state (according to a recurrence threshold $\varepsilon$ ) of a dynamic system repeat along time [28].

To quantify the HVR recurrences for each period, we choose six factors displayed by the Recurrent Plots (RPs) that are $[26,28]$ :

- Recurrence Rate (RR) - quantifies the density of recurrences.

- Determinism (DTM) - quantifies the density of the recurrence time intervals.

- Maximum Diagonal Line Length (Lmax) - indicates the maximum length of a time recurrence

- Shannon Entropy (ENTR) - quantifies the complexity of the interval recurrences.

- Laminarity $(L)$ - quantifies the percentage of fixed events in a given time interval of recurrences.

- Trapping Time (TT) - estimates the mean value of fixed events in a given time interval of recurrences.

\section{E. Statistical Analysis}

All data were submitted to the Kolmogorov-Smirnov normality test for data verification [29]. Since the samples were not considered as following a normal distribution, nonparametric tests were used.

Group differences were assessed using Kruskal-Wallis test, followed by the Sîdák post-hoc test [30]. For two-sample comparisons, we used the Mann-Whitney test. Additionally, it was used Bartlet test to compare differences in variances [31]. Each test was repeated 100 times to evaluate the consistency and time-series stationarity in relation to each epoch $1 \mathrm{~min}, 2 \mathrm{~min}, 4 \mathrm{~min}$ and $6 \mathrm{~min}$. The significance level for all statistical analyses was established as $\alpha=0.05$. Principal Component Analyis (PCA) [32] was applied to explore the differences between RQA coefficients and PM features obtained from rMSSD for the fragments of all lengths.

\section{RESULTS AND DISCUSSIONS}

In Fig. 1, a fragment of HRV signals based on R-R successive measures from spontaneous breathing and controlled breathing is presented.
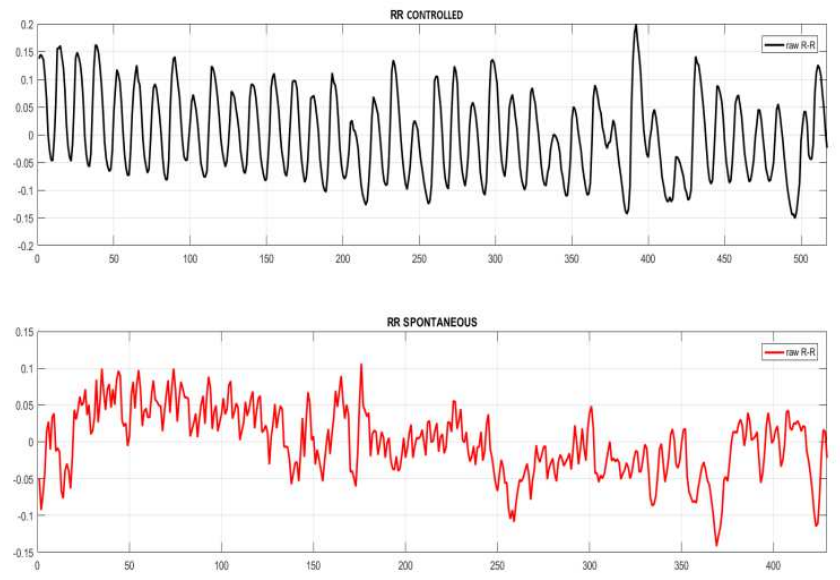

Fig. 1 Example of R-R measures from controlled breathing (in black) and spontaneous breathing (in red)

After that the recurrences from controlled and spontaneous breathing were plotted and presented in Fig. 2. It is possible to notice the amount of differences in the phase space and in the RP of each signal that the attractors can capture. While the attractor and the RP of the signals with controlled breathing present the most regular and deterministic patterns, the attractor and the RP of spontaneous breathing have classic patterns of coupled systems, with tendencies of chaoticity and stochasticity.
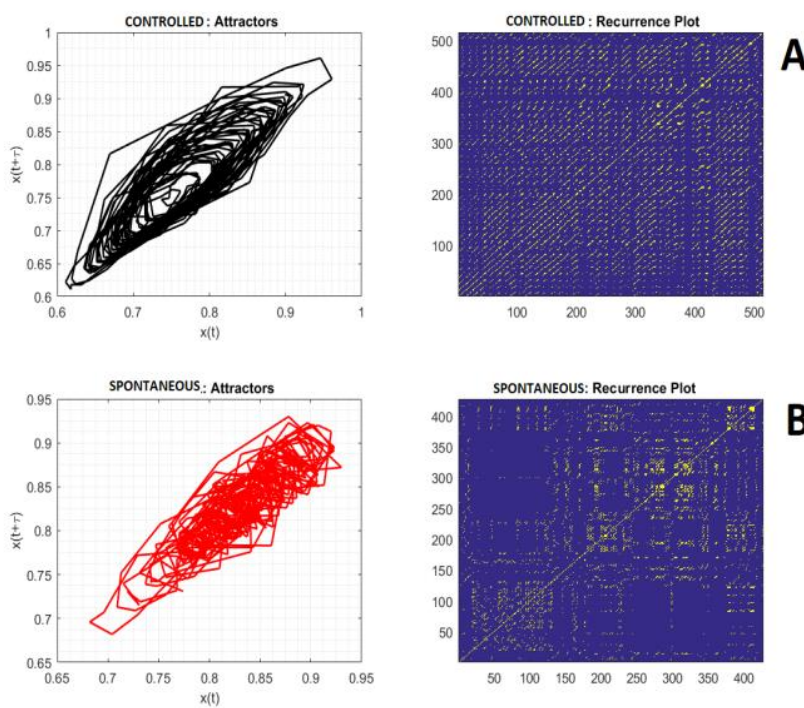

Fig. 2 Visualization of the recurrences of HVR signals from controlled and spontaneous breathing. (A) Attractor of a subject with controlled breathing and its RP, showing deterministic patterns. (B) Attractor of the same subject with spontaneous breathing and its RP, showing chaotic patterns.

Fig. 3 shows examples of Poincare Maps for each period of analysis considered (6 min, $3 \mathrm{~min}, 2 \mathrm{~min}$ and $1 \mathrm{~min}$ ). It is 
possible to see how the reduction of the time length of the R$\mathrm{R}$ signals, contributes for a clear the visualization of the Poincare map. This could also impact on the quality of any analysis that is going to be carried out. The distribution of the signals with controlled breathing, despite being more regular, have greater amplitudes, which is also possible to notice in the PM.

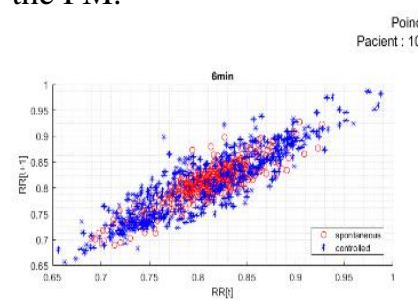

$$
\begin{aligned}
& \text { Poincaré Map } \\
& \text { Pacient : 10/Amostra : } 30
\end{aligned}
$$
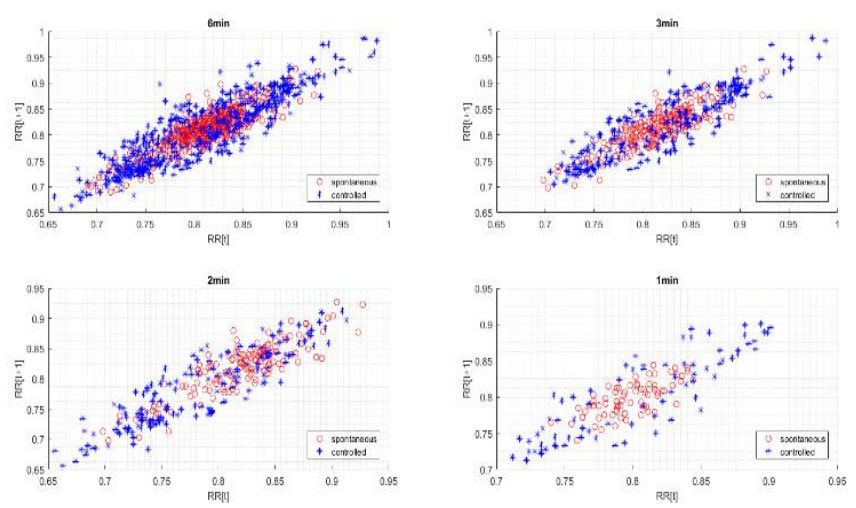

Fig. 3 Poincare maps for the periods of analyses (6 min, $3 \mathrm{~min}, 2 \mathrm{~min}$ and $1 \mathrm{~min}$ )

The following step was to verify if the coefficients obtained by the PMs and the RMSSD from spontaneous and controlled breathing R-R signals were significatively different (Fig. 4). For this analysis, it was compared the data obtained for each period of interest ( $1 \mathrm{~min}, 2 \mathrm{~min}, 4 \mathrm{~min}$ and $6 \mathrm{~min})$. Hundred fragments were randomly selected with reposition throughout each record of each participating subject.

Above each plot it is possible to see the proportion in which the 100 comparisons analyzed (from the 100 random selections), showed statistical difference, in each test. For the first analysis of SD1, for example, from 100 comparisons, none was significant between the different time lengths and only 19 comparisons showed any significance between the 4time lengths of interest).

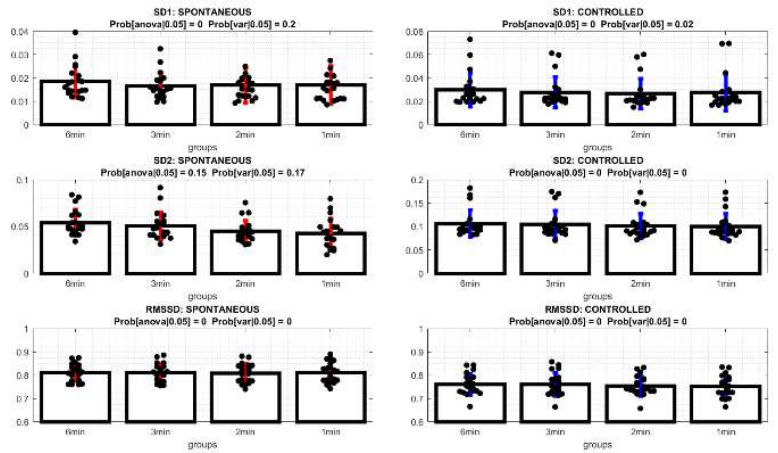

Fig. 4 Statistical Analyses considering coefficients from PMs and rMSSD of the R-R signals
Then, it was performed a comparative analysis between mean values and variance from the RQA coefficients for controlled and spontaneous breathing (Fig.5). The first 6 plots exemplify the 100 comparisons made using Kruskal-Wallis (with Sîdák, posthoc) for comparisons between means and Bartlet for comparisons between variances. The title of each plot also shows the number of times, proportionally, each comparison was significant, in the 100 comparisons performed. For both controlled and spontaneous breathing, the Lmax and ENT coefficients were the most relevant in the all comparisons.
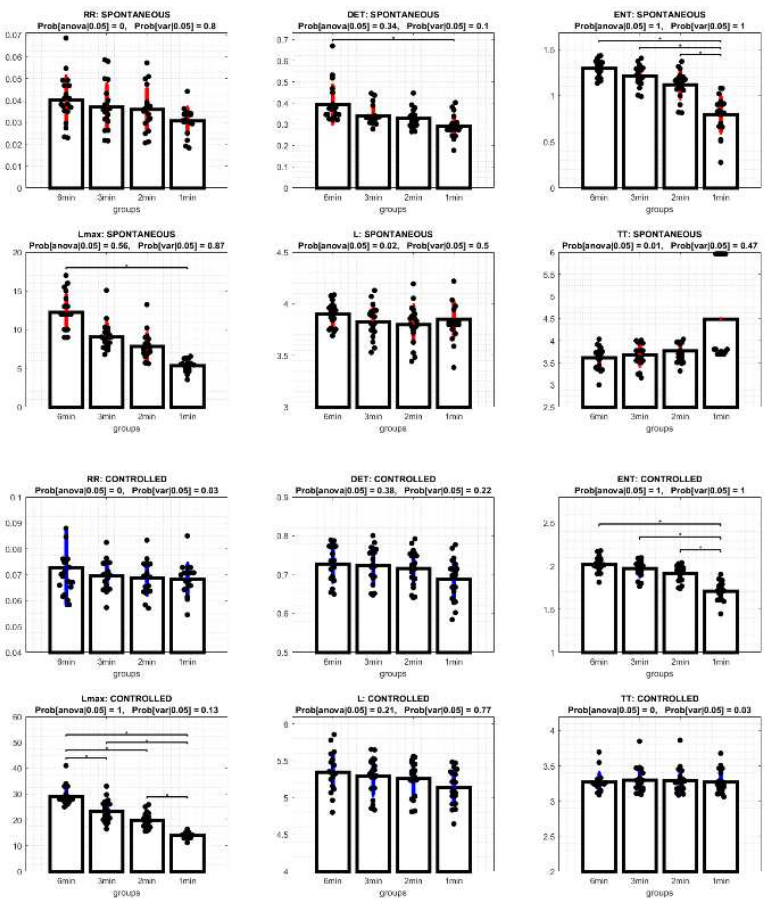

Fig. 5 Comparative statistical analysis between means and variance from the RQA coefficients for controlled and spontaneous breathing.

Finally, it was plotted a Principal Component Analysis using coefficients PM+rMSSD and the coefficients of RQA for R-R signals, for controlled and spontaneous breathing. The first row of the plots shows that using only the coefficients SD1, SD2 and RMSSD as features in the PCA, it is impossible to make a distinction between the four periods of interest. This first result may precisely lead to the misleading conclusion that there are no differences between the four sections. However, when using the RQA coefficients as features of the PCA, the difference in each of the four analyzed sections is evidenced. For the signals of controlled breathing it is possible to observe the large difference in the average of the clusters and their spread, especially between the clusters associated with the $1 \mathrm{~min}$ and 6 min periods. With the RQA, it is also possible to visualize a separation by blocks between 
each period, distinguishing them completely, even in a situation of spontaneous breathing.
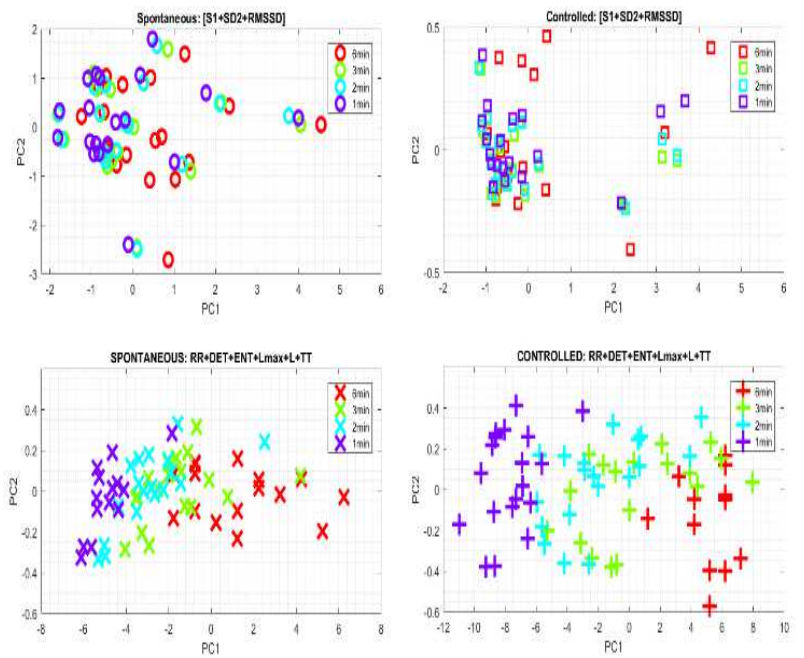

Fig. 6 Principal Component Analysis using coefficients PM+rMSSD and the coefficients of RQA for R-R signals, for controlled and spontaneous breathing.

\section{iv. Conclusions}

Most standard techniques, that estimates temporal characteristics (or in the frequency domain) of tachogram (R-R signals) to assess heart rate variability are quite promising and, in many situations, efficient. Although, it is necessary to be careful with the conclusions taken from these indices since analyzes bias may occur because of the lack of a standard criterion for a minimum registration time for the estimation of these factors. As the cardiorespiratory system is a non-linear system, with characteristics of stochasticity and chaoticity, it is possible that standard techniques are not able to detect important non-linear fluctuations, which are added up in larger periods, and are extremely important for in HRV analysis. This is evident when applying techniques capable of detecting these non-linear fluctuations such as RQA. Using the RQA, we were able to show the discrepancy between the four segments of interest, both on mean and in variance, explained in the analysis of main components.
As a limitation of this study, it is important to mention that cardiac autonomic tone adaptability to breathing frequency can vary for each individual. This is a relevant bias to be considered when using a controlled breathing rhythm. In slow paced respiratory frequency protocols, the time difference of automatic cardiac tone mobilization between the participants can influence on the replication of short time epochs. Another important limitation of controlled breathing is that the cardiac autonomic tone adaptation to the body posture can generate non-stationary artifacts that impair the replication of ultra-short HRV epochs. Thus, to obtain replicable epochs to ultra-short HRV analysis, it is necessary a period of adaptation to cardiac autonomic tone to the posture.

\section{AcKNOWLEDGMENT}

This work was supported by PRONEX Program (Programa de Núcleos de Excelência - NENASC Project) of FAPESC-CNPq-MS, Santa Catarina Brazil (process number 56802/2010). RW is a Researcher Fellow from CNPq (Brazilian Council for Scientific and Technologic Development, Brazil) and HMM is supported by CAPES/DS scholarship. We also would like to thank for the Call of the Support of Assistive Technology Projects - TA2016/CNPq, process number: 442563/2016-7

\section{CONFLICT OF INTEREST}

The authors declare that they have no conflict of interest.

\section{REFERENCES}

1. Task Force of the European Society of Cardiology and the North American Society of Pacing and Electrophysiology (1996) Heart rate variability: Standards of measurement, physiological interpretation, and clinical use. Circulation 93(5): 1043-1065 DOI 10.1161/01.CIR.93.5.1043

2. Billman G E, Huikuri H V, Sacha J et al (2015) An introduction to heart rate variability: methodological considerations and clinical applications. Frontiers in Physiology 6:2013-2015 DOI 10.3389/fphys.201400177

3. Laborde S, Mosley E, Thayer J F (2017) Heart rate variability and cardiac vagal tone in psychophysiological research - recommendations for experiment planning, data analysis, and data reporting. Front. Psychol. 8: 118 DOI 10.3389/fpsyg.2017.00213

4. Arroyo-Carmona R E, López-Serrano A L, Albarado-Ibañez A et al. (2016) Heart rate variability as early biomarker for the evaluation of diabetes mellitus progress. Journal of Diabetes Research 2016:1-8. DOI 10.1155/2016/8483537

5. Kleiger R E, Stein P K, Bigger J T (2005) Heart rate variability: measurement and clinical utility. Ann Noninvasive Electrocardiol. 10(1):88-101 DOI 10.1111/j.1542-474X.2005.10101.x 
6. Thayer J F, Lane R D (2000) A model of neurovisceral integration in emotion regulation and dysregulation. J Affect Disord. 61(3): 201-216 DOI 10.1016/s0165-0327(00)00338-4

7. Thayer J F, Yamamoto S S, Brosschot J F (2010) The relationship of autonomic imbalance, heart rate variability and cardiovascular disease risk factors. Int J Cardiol. 141(2): 122-131. DOI 10.1016/j.ijcard.2009.09.543

8. Yadav R L, Yadav P K, Yadav L K et al (2017) Association between obesity and heart rate variability indices: an intuition toward cardiac autonomic alteration - a risk of CVD. Diabetes Metab Syndr Obes. 10: 57-64. DOI 10.2147/DMSO.S123935

9. Beauchaine T P, Thayer J F (2015) Heart rate variability as a transdiagnostic biomarker of psychopathology. Int J Psychophysiol. 98(2 pt 2): 338-350 DOI 10.1016/j.ijpsycho.2015.08.004

10. Thayer J F, Ahs F, Fredrikson M, et al (2012) A meta-analysis of heart rate variability and neuroimaging studies: Implications for heart rate variability as a marker of stress and health. Neurosci Biobehav Rev. 36(2): 747-756 DOI 10.1016/j.neubiorev.2011.11.009

11. Thayer J F, Lane R D (2009). Claude Bernard and the heart-brain connection: further elaboration of a model of neurovisceral integration. Neurosci Biobehav Rev. 33(2): 81-88 DOI 10.1016/j.neubiorev.2008.08.004

12. Sá J C F, Costa E C, Silva E et al (2013) Heart rate variability as a method of assessing the autonomic nervous system in polycystic ovary syndrome. Rev Bras Ginecol Obstet 35(9):421-426 DOI 10.1590/S010072032013000900007

12. Giles D, Draper N, Neil W (2016) Validity of the Polar V800 heart rate monitor to measure RR intervals at rest. European Journal of Applied Physiology 116(3): 563-571 DOI 10.1007/s00421-015-3303-9

13. Nussinovitch U, Elishkevitz K P, Katz K et al (2011) Reliability of ultra-short ECG indices for heart rate variability. Ann Noninvasive Electrocardiol. 16(2):117-122 DOI 10.1111/j.1542-474X.2011.00417.x

14. Melo H M, Martins T C, Nascimento L M et al (2018) Ultra-short heart rate variability recording reliability: The effect of controlled paced breathing. Ann Noninvasive Electrocardiol. 4(4):1-9 DOI 10.1111/anec. 12565

15. Munoz M L, Van Roon A, Riese H et al (2015) Validity of (Ultra-) Short recordings for heart rate variability measurements. PLoS ONE, 10(9): 1-15 DOI 10.1371/journal.pone.0138921

16. Esco M R, Flatt A A (2014) Ultra-short-term heart rate variability indexes at rest and post-exercise in athletes: Evaluating the agreement with accepted recommendations. J Sports Sci Med. 13(3): 535-541

17. Nakamura F Y, Flatt A A, Pereira L A et al (2015) Ultra-Short-Term heart rate variability is sensitive to training effects in team sports players. $\mathrm{J}$ Sports Sci Med 14(3): 602-605

18. Melo H M, Hoeller A A, Walz R et al (2019) Resting cardiac vagal tone is associated with long-term frustration level of mental workload: ultrashort term recording reliability. Appl Psychophysiol Biofeedback 45: 1-9 DOI 10.1007/s10484-019-09445-z

19. Salahuddin L., Cho J, Jeong M G et al (2007) Ultra short term analysis of heart rate variability for monitoring mental stress in mobile settings. Conf Proc IEEE Eng Med Biol Soc. 2007, 2007, pp. 4656-4659 DOI 10.1109/IEMBS.2007.4353378

20. Nussinovitch U, Cohen O, Kaminer K et al (2012) Evaluating reliability of ultra-short ECG indices of heart rate variability in diabetes mellitus patients. Journal of Diabetes and Its Complications 26(5):450-453 DOI 10.1016/j.jdiacomp.2012.05.001

21. Pecchia L, Castaldo R, Montesinos L et al (2018) Are ultra-short heart rate variability features good surrogates of short-term ones? State-ofthe-art review and recommendations. Healthc Technol Lett. 5(3): 94-100 DOI 10.1049/htl.2017.0090

22. Strumillo P, Ruta J (2002) Poincare mapping for detecting abnormal dynamics of cardiac repolarization. IEEE Engineering in Medicine and Biology Magazine 21(1): 62-65 DOI 10.1109/51.993195.

23. Che-Hao H, Ming-Ya T, Go-Shine H, Tso-Chou L, Kuen-Pao C, Shung-Tai H, Liang-Yu S, Chi-Yuan L (2012) Poincaré plot indexes of heart rate variability detect dynamic autonomic modulation during general anesthesia induction. Acta Anaesthesiol Taiwan. 50(1): 12-18. DOI 10.1016/j.aat.2012.03.002

24. Arcentales A, Giraldo B F, Caminal P et al (2011) Recurrence quantification analysis of heart rate variability and respiratory flow series in patients on weaning trials, Conf Proc IEEE Eng Med Biol Soc. 2011, pp 27242727 DOI 10.1109/IEMBS.2011.6090747

25. Trunkvalterova Z, Javorka M, Tonhajzerova I et al (2007) Recurrence Quantification Analysis of Heart Rate Dynamics in Young Patients with Diabetes Mellitus, IFMBE Proc. vol. 16, 11th Mediterranean Conference on Medical and Biomedical Engineering and Computing 2007. Springer, Berlin, Heidelberg

26. Webber C H, Junior N M (2015) Recurrence Quantification Analysis. Springer, Switzerland

27. Brennan M, Palaniswami M, Kamen P (2001). Do existing measures of Poincare plot geometry reflect nonlinear features of heart rate variability? IEEE Transactions on Biomedical Engineering 48(11): 1342-1347. DOI $10.1109 / 10.959330$

28. Marwan N, Romano M C, Thiel M et al (2007) Recurrence plots for the analysis of complex systems. Physics Reports 438(5): 237-329. DOI 10.1016/j.physrep.2006.11.001

29. Frank J, Massey Jr (1951) The Kolmogorov-Smirnov Test for Goodness of Fit. Journal of the American Statistical Association 46(253): 68-78, DOI 10.1080/01621459.1951.10500769

30. Kramer C Y (1956) Extension of Multiple Range Tests to Group Means with Unequal Numbers of Replications. Biometrics 12(3): 307-310 DOI $10.2307 / 3001469$

31. McHugh M L (2013) The chi-square test of independence. Biochem Med (Zagreb) 23(2):143-149 DOI 10.11613/bm.2013.018

32. Jolliffe I T, Cadima J (2016) Principal component analysis: a review and recent developments Philos Trans A Math Phys Eng Sci. 374(2065): :20150202 DOI 10.1098/rsta.2015.0202

Enter the information of the corresponding author:

Author: Mariana Cardoso Melo

Institute: Universidade Federal de São Paulo

Street: Botucatu Street, 862

City: São Paulo

Country: Brazil

Email:mcmelo@unifesp.br 